\title{
Design of Low-loss Arrayed Waveguide Gratings for Applications in Integrated Raman Spectroscopy
}

\author{
N. Ismail ${ }^{1}$, I. Akca ${ }^{1}$, A. C. Baclig ${ }^{2}$, P. J. Caspers ${ }^{2}$, F. Sun ${ }^{1}$, K. Wörhoff ${ }^{1}$, R. M. de Ridder ${ }^{1}$, M. Pollnau ${ }^{1}$, and \\ A. Driessen ${ }^{1}$ \\ ${ }^{1}$ Integrated Optical Micro Systems Group, MESA+ Institute for Nanotechnology, University of Twente, \\ P.O. Box 217, 7500 AE Enschede, The Netherlands \\ ${ }^{2}$ Center for Optical Diagnostics and Therapy, Department of Dermatology, Erasmus MC Rotterdam, The Netherlands \\ n.ismail@ewi.utwente.nl
}

\begin{abstract}
An integrated low-loss arrayed waveguide grating is designed for Raman spectroscopy of the human skin. The device layout targets spectral analysis of Raman-scattered light for in vivo determination of water concentration in the stratum corneum.

(C)2010 Optical Society of America

OCIS codes: (300.6450) Spectroscopy, Raman; (130.7408) Wavelength filtering devices; (170.6510) Tissue diagnostics;
\end{abstract}

\section{Introduction}

In vivo confocal Raman spectroscopy of the skin was introduced in 1998 [1]. This method provides a possibility of accurately controlling the skin region from which the Raman signal is detected. Our challenge is to realize an integrated, low-cost, compact, hand-held optical device for in vivo confocal Raman spectroscopy of the skin, specifically the detection of water concentration in the stratum corneum. Water concentration can be measured in terms of ratios between the detected signals in the water, lipid, and protein bands. The Raman spectrum of the skin is shown in Fig. 1(a) for different values of water concentration, together with the required bands.

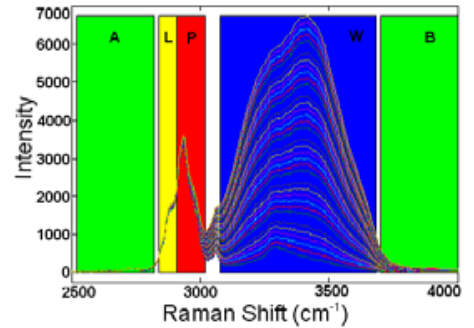

(a)

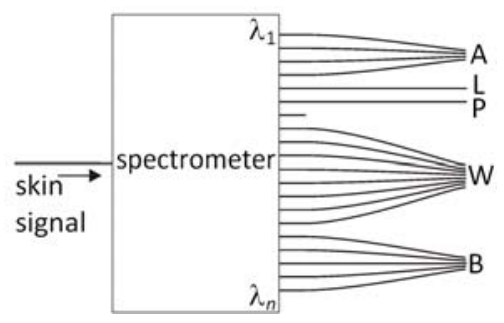

(b)

Fig. 1. a) Raman spectrum of the skin for different values of water concentration, with bands A and B, background; L, lipid; P, protein; and W, water. b) Each group of output channels of the on-chip spectrometer transmitting light at multiple wavelengths associated with the same band is brought to a single detector (indicated by the labels A, L, P, W, and B, respectively).

\section{Arrayed waveguide grating for wavelength separation in the Raman spectral region}

We have designed an arrayed waveguide grating (AWG) spectrometer on a thermally oxidized silicon wafer using low-birefringence silicon oxynitride ( $\mathrm{SiON}$ ) waveguides with $\mathrm{SiO}_{2}$ cladding. The waveguides have a cross-section of $1.4 \mu \mathrm{m} \times 0.52 \mu \mathrm{m}$ and a core refractive index for TE polarization of 1.509 at $830 \mathrm{~nm}$. The material birefringence is $\Delta n_{\mathrm{TM} \text {-TE }}=2.1 \times 10^{-3}$ for $\mathrm{SiON}$ and $1.0 \times 10^{-3}$ for $\mathrm{SiO}_{2}$ [2]. As verified experimentally, the guiding material does not generate luminescence in the Raman wavelength range when excited with 671-nm laser light (excitation wavelength for the skin application). The requirements for wavelength selection are shown schematically in Fig. 1. The Raman shift is indicated in units of $\mathrm{cm}^{-1}$, which for our excitation wavelength translates into a wavelength range from 800 $\mathrm{nm}$ to $920 \mathrm{~nm}$. The resolution of the device must be large enough to separate the lipid and protein peaks; taking into account that the lipid band is $5 \mathrm{~nm}$ wide and that in this wavelength region the Raman signal is weaker than in the $\mathrm{P}$ and W bands, we choose the central wavelength of the AWG at the center of the lipid band (831 nm) and an output channel spacing of $5 \mathrm{~nm}$. In this way we guarantee the lowest insertion loss for the lipid peak. To cover the entire Raman signal a minimum free spectral range of $120 \mathrm{~nm}$ is required; the best choice of grating order is $m=3$, resulting in 55 output channels. Other values of $m$ would result in the (backscattered) laser wavelength overlapping with or falling close to one of the Raman channels. With our choice of channel spacing we can place only one channel in each of the L and P bands, while more than one channel can be placed to collect the background and the water signals. Each group of channels is brought to a single detector as shown in Fig. 1(b). The requirement of a low order, $m=3$, makes it necessary to use a broadband s-shaped AWG layout; with conventional horse-shoe geometry it is impossible to interconnect the arrayed waveguides to the free propagation regions with correct angular relations. 
Making use of low-birefringence waveguides (zero birefringence in the center of the Raman signal range and \pm 1 $\times 10^{-4}$ at the edges) for the array is required for the AWG to be polarization insensitive [3]. The simulation results for TE polarization are shown in Fig. 2(a). For TM polarization we observed negligible shifts of $0.01 \mathrm{~nm}$ for the central channels, and $0.03 \mathrm{~nm}$ in the $\mathrm{W}$ band, see inset in Fig. 2(a). Very low insertion losses are achieved, ranging from 1.1 $\mathrm{dB}$ for the central channel up to a maximum of $2 \mathrm{~dB}$ for the outermost channels. Adjacent crosstalk is $-10.5 \mathrm{~dB}$, while non-adjacent crosstalk is $-37 \mathrm{~dB}$ for the central channels and increases up to $-27 \mathrm{~dB}$ at higher wavelengths.

\section{Novel layout for loss reduction}

Furthermore, we introduce improvements in the geometrical layout of the device in order to reduce the losses. The Raman signal may be up to $10^{8}$ times weaker than the backscattered laser and fluorescence light; hence the reduction of losses in the device is an important part of the design. For this reason we modified the standard anti-symmetric broadband layout [4] to reduce the main sources of loss, which are intrinsically part of the conventional geometry. We identify these in straight-to-bend waveguide transition losses [5] and systematic phase errors due to the use of different bends for the arrayed waveguides. A method for reducing the systematic phase errors has been reported in [6], in which bends with constant bending radius (but different length) were used in all the arrayed waveguides. In our design we aimed to completely eliminate these phase errors by using identical bends in all arrayed waveguides. We make use of two bend types (A and B) which are used as building blocks in all the arrayed waveguides. All arrayed waveguides are composed of two bends of type A and $N-1$ bends of type B, where $N$ is the number of arrayed waveguides. In this way the phase of light propagating in the array is affected in the same way by the bends, while the optical path difference is introduced only by straight sections. Since this approach is independent of the geometry of the bend that is being used, we replaced all circular bends with cosine bends in which the transition from straight to bend is gradual and can be designed to be adiabatic. An example of the AWG layout is shown in Fig. 2(b).

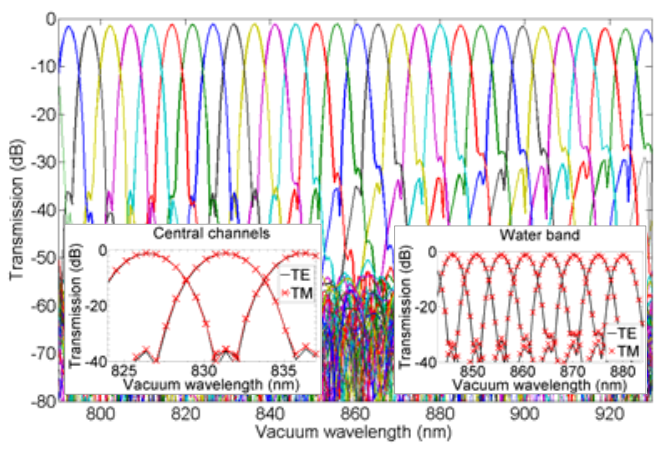

(a)

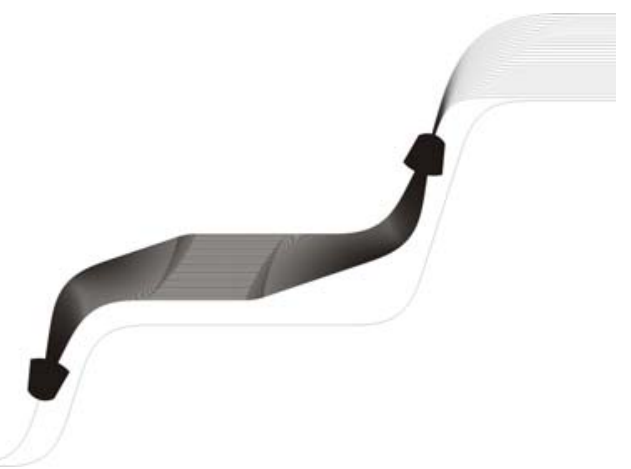

(b)

Fig. 2. a) Wavelength response of the AWG for the skin application for TE and TM polarizations; b) example layout of our structure which makes use of identical bends in all the arrayed waveguides

\section{Conclusions}

We have discussed the design issues related to an integrated device for wavelength separation of Raman signals collected from the skin in the near IR. The device presents improvements in the geometrical layout aimed at reducing losses and is designed to be polarization insensitive. We have fabricated devices based on SiON technology according to this design and their optical characterization is currently under way.

Financial support from the Dutch IOP Photonic Devices (Senter-Novem and STW) is acknowledged.

\section{References}

[1] P. J. Caspers, G. W. Lucassen, R. Wolthuis, H. A. Bruining, and G. J. Puppels, "In vitro and in vivo Raman spectroscopy of human skin," Biospectroscopy 4, S31-S39 (1998).

[2] K. Wörhoff, L. T. H. Hilderink, A. Driessen, and P. V. Lambeck, "Silicon oxynitride: a versatile material for integrated optics application," J. Electrochem. Soc. 149, 85-91 (2002).

[3] M. K. Smit and C. v. Dam, "PHASAR-based WDM-devices: Principles, design and applications," J. Select. Topics Quantum Electron. 2, 236-250 (1996).

[4] R. Adar, C. H. Henry, C. Dragone, R. C. Kistler, and M. A. Milbrodt, "Broad-band array multiplexers made with silica waveguides on silicon," J. Lightwave Technol. 11, 212-219 (1993).

[5] M. K. Smit, "Progress in AWG design and technology," in IEEE/LEOS Workshop on Fibers and Optical Passive Components (IEEE/LEOS Italian Chapter, Palermo, 2005), pp. 26-31.

[6] F. M. Soares, W. Jiang, N. K. Fontaine, S. W. Seo, J. H. Baek, R. G. Broeke, J. Cao, K. Okamoto, F. Olsson, S. Lourdudoss, and S. J. B. Yoo, "InPbased arrayed-waveguide grating with a channel spacing of $10 \mathrm{GHz}$," in National Fiber Optic Engineers Conference (Optical Society of America, Washington DC, 2008), paper JThA23. 